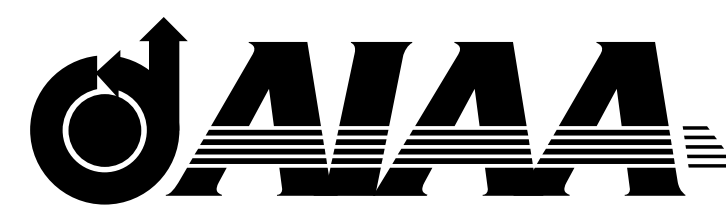

\title{
AIAA 2000-4858 \\ IMPLEMENTING AUTOMATIC \\ DESIGN OPTIMISATION IN AN INTERACTIVE ENVIRONMENT
}

Hassan Ugail, Malcolm I. G. Bloor, and Michael J. Wilson

University of Leeds, Leeds LS2 9JT, United Kingdom

\section{8th AIAA/USAF/NASA/ISSMO Symposium on Multidisciplinary Analysis and Optimization September 6-8, 2000/Long Beach, California}

For permission to copy or republish, contact the American Institute of Aeronautics and Astronautics 1801 Alexander Bell Drive, Suite 500, Reston, VA 20191-4344 


\title{
IMPLEMENTING AUTOMATIC DESIGN OPTIMISATION IN AN INTERACTIVE ENVIRONMENT
}

\author{
Hassan Ugail, Malcolm I. G. Bloor; and Michael J. Wilson ${ }^{\ddagger}$ \\ University of Leeds, Leeds LS2 9JT, United Kingdom
}

\begin{abstract}
In this paper we consider shape optimization using a geometry parameterization that can be interactively specified by a user sitting at a workstation. The shape geometry is created using a boundary-value approach known as PDE method which uses information at the boundaries (edges) of the surface to define and manipulate the shape. Using this design approach enables one to parameterize complex geometric shapes using a small set of design parameters. The variation of the chosen design parameters enable to create alternative configurations of the same design and a suitable optimization scheme then allows to identify an optimal design with respect to a chosen merit function. In this paper the method of Simulated Annealing serves as the optimization scheme. Thus, we show how optimal shapes which are of practical significance can be automatically designed in an interactive setting.
\end{abstract}

\section{Nomenclature}

$E^{3} \quad$ euclidean 3 -space

$x, y, z \quad$ cartesian coordinates

$\Omega \quad$ domain of solution in $\Re^{2}$

$\underline{X}(u, v) \quad$ vector of Cartesian coordinates of the surface points

$\underline{X}_{u} \quad$ derivative vector

a smoothing parameter

$p_{i} \quad i$ th positional boundary curve

$d_{i} \quad i$ th derivative boundary curve

$c_{k P i} \quad(k=1,2$ and $i=x, y, z)$, parameterization $P$ of a boundary curve $c$

$\sigma^{i} \quad$ shear stress at a point $i$ of the surface

\section{Introduction}

A typical requirement in practical engineering design is to minimize or maximize an objective function without violating a set of constraints. In order to improve a design by applying methods of computational optimization, it is necessary to express the design objective and constraints of the optimization problem by an appropriate mathematical formulation. A general formulation of the optimization problem can be written as

$$
\begin{array}{r}
\min \left\{f(\underline{x}) \mid \underline{x}_{l} \leq \underline{x} \leq \underline{x}_{u} ; \underline{g}(\underline{x})=0 ;\right. \\
\underline{h}(\underline{x}) \leq 0\}, \quad \underline{x} \in \Re^{n},
\end{array}
$$

\footnotetext{
${ }^{*}$ Research Fellow, Department of Applied Mathematics.

${ }^{\dagger}$ Professor, Department of Applied Mathematics.

¥Professor, Department of Applied Mathematics.

Copyright (c) 2000 by the American Institute of Aeronautics and Astronautics, Inc. All rights reserved.
}

\begin{abstract}
with
$f \quad$ the objective function;

$\underline{x} \quad$ vector of $n$ design variables;

$g \quad$ vector of $p$ equality constraints;

$\underline{h} \quad$ vector of $q$ inequality constraints;

$\underline{x}_{l}$ and $\underline{x}_{u} \quad$ lower and upper bounds for the design variables.

The design variables and the constraints form the feasible design space
\end{abstract}

$$
\underline{x} \in R^{n} \mid \underline{x}_{l} \leq \underline{x} \leq \underline{x}_{u} ; \underline{g}(\underline{x})=0 ; \underline{h}(\underline{x}) \leq 0,
$$

in which the trial design should lie.

Coming up with appropriate formulations of the design objective and constraints of the optimization problem in an engineering application is not always a trivial task. According to Cohen, ${ }^{4}$ due to the complex nature of most engineering problems the choice of the right objective function requires experience and a fundamental understanding of the design objectives. Furthermore, not all constraints can be easily formulated in a mathematically correct way for optimization.

In the past, many different approaches have been taken to perform the actual optimization. For example, Zeinkiewicz and Campbell ${ }^{19}$ considered optimization in terms of successive linear programming problems. The constraints and the objective function are linearized about the current design variable values and this simplified problem is solved. The result is taken as the new starting design and the process is repeated until no further improvements can be made. This method has the advantage of making use of the efficient linear programming algorithms that are available. 
An alternative approach was taken by Francavilla et $a l^{6}$ and Yoo et al ${ }^{18}$ where a search algorithm based on the design sensitivities is used to solve the full non-linear problem, again in an iterative manner. For this type of approach to optimization, a point worth noting is that the existence of a fundamental difficulty which arises in the initial formulation of the optimization problem. For example, in the above examples, the design variables were effectively the Cartesian coordinates of points that boundary curves of a particular form defining the geometry of the shape were required to pass through. Thus, there is lack of an appropriate parameterization of the geometry of the shape.

In their attempts to simplify the problem of shape optimization, Kristensen and Madsen ${ }^{9}$ described the boundary of the geometric model, in their case the shape of an engine bearing cap, as a weighted sum of certain specified functions, the weights being taken as the design variables. Furthermore, Yao and $\mathrm{Choi}^{17}$ adopted the surface parameterization developed by Bézier for shape optimization. However, although the surface formulation involved many design freedoms, in the problem considered the surface was severely restricted which left only a few design variables. The surface was constrained to bend in only one direction, effectively being formed by a planar curve swept without distortion along a straight line perpendicular to its plane, and the points defining its shape only allowed to move in one of three possible directions.

It has been noted by several authors in the shape optimization literature that the most important aspect of shape optimization is the choice of the design variables to be used and how the boundary shape is parameterized in terms of these design variables. ${ }^{6,7}$ Choosing too many variables will considerably complicate the design problem with severe implications on the computational time required, and having too few variables may result in only trivial solutions being obtained. ${ }^{6,7}$ It is therefore a basic requirement that a wide range of shapes (which can be defined by a relatively small number of parameters) are accessible to the method of optimization used.

In this paper, we discuss how a method for geometry parameterization known as the PDE method can be used to set up a practical optimization problem. The PDE method can completely define the geometry of a shape in terms of the boundaries (edges) of the surface patch along with a small set of design parameters. Thus, using the PDE method it is possible to formulate the design optimization problem by using a small number of design parameters which can be chosen in an in- tuitive fashion making the optimization problem less complicated. Furthermore, the choice of fewer design parameters implies that the numerical calculations required to perform the optimization can be carried out more efficiently.

In the rest of this paper we discuss how the PDE surfaces can be created and parameterized interactively. Furthermore, we show how the parameterized PDE model can be used to setup the automatic optimization enabling us to obtain practically significant optimal shapes with respect to a chosen merit function.

\section{The PDE Method and Geometry Generation}

In geometric design, it is common practice to define curves and surfaces using some form which represents the surface parametrically. Thus, surfaces are defined in terms of two parameters $u$ and $v$ so that any point on the surface $\underline{X}$ is given by an expression of the form:

$$
\underline{X}=\underline{X}(u, v) .
$$

Equation (3) can be viewed as a mapping from a domain $\Omega$ in the $(u, v)$ parameter space to $\mathrm{Eu}-$ clidean 3 -space. In the case of the PDE method this mapping is defined as a partial differential operator:

$$
L_{u v}^{m}(\underline{X})=\underline{F}(u, v),
$$

where the partial differential operator $L$ is of degree $m$. Thus, effectively, surface design is treated as an appropriately posed boundary-value problem with boundary conditions imposed on $\partial \Omega$, the boundary of $\Omega$. The partial differential operator $L$ is usually taken to be that of elliptic type and the degree $m$ of this operator depends on the level of surface control and continuity required at the boundaries of the surface. The function $\underline{F}(u, v)$ is included for completeness and is generally taken to be zero.

The PDE method has been discussed before by a number of different references, e.g. Vida et al, ${ }^{16}$ Bloor and Wilson ${ }^{1}$ and Bloor and Wilson. ${ }^{2}$ It has been shown how surfaces satisfying a wide range of functional requirements can be created by a suitable choice of the boundary conditions and appropriate values for the various design parameters associated with the method. ${ }^{5,14,15}$

\section{Interactive Design}

For the work described here, and for the majority of previous work carried out using the PDE method described elsewhere, the PDE chosen is of 

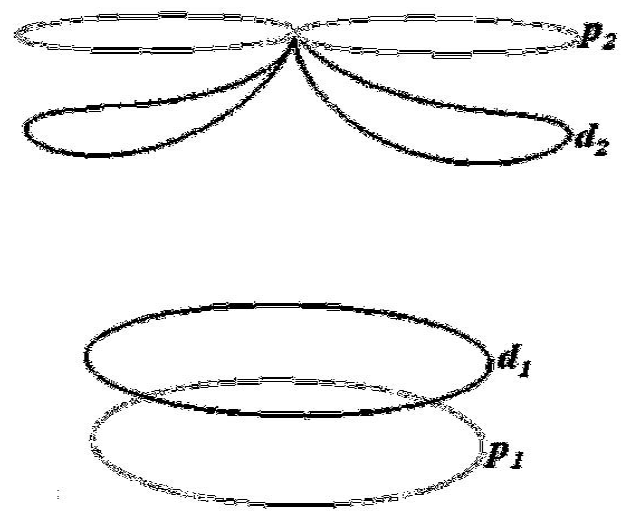

(a)

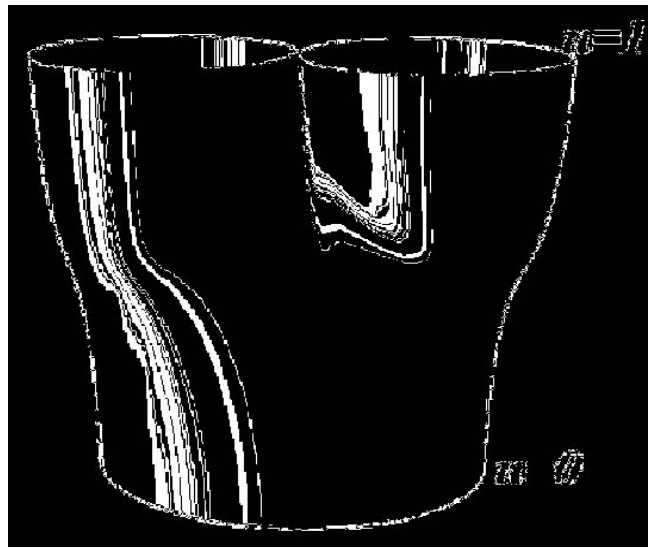

(b)

Fig. 1 Typical PDE surface. (a) The boundary curves. (b) The corresponding PDE surface patch

the form:

$$
\left(\frac{\partial^{2}}{\partial u^{2}}+a^{2} \frac{\partial^{2}}{\partial v^{2}}\right)^{2} \underline{X}(u, v)=0
$$

where the condition on the function $\underline{X}(u, v)$ and its normal derivatives $\frac{\partial \underline{X}}{\partial n}$ can be imposed at the edges of the surface patch. The parameter $a$ is a special design parameter which controls the relative smoothing of the surface in the $u$ and $v$ directions. ${ }^{2}$ For periodic boundary conditions (e.g. $0 \leq u \leq 1,0 \leq v \leq 2 \pi)$, a pseudo-spectral method has been developed for the solution of equation (5) which allows $\underline{X}(u, v)$ to be expressed in closed form. ${ }^{3}$

As far as interactive design is concerned, the boundary conditions are usually defined in terms of curves in 3-space. For example, Fig. 1 shows a typical set of boundary curves and the corresponding PDE surface showing the port of a bifurcated transfer port of a 2-stroke engine. Here the value of $a$ was taken to be 1.0100 . Note that the curves marked $p_{1}$ and $p_{2}$ correspond to the boundary conditions on the function $\underline{X}(u, v)$. A vector field corresponding to the difference between the points on the curves marked $p_{1}$ and $p_{2}$ and those marked $d_{1}$ and $d_{2}$ respectively, corresponds to the conditions on the function $\frac{\partial X}{\partial n}$ such that

$$
\frac{\partial \underline{X}}{\partial n}=[\underline{p}(v)-\underline{d}(v)] s,
$$

where $s$ is a scalar. The conditions defined by $p_{1}$, $p_{2}$ and $d_{1}, d_{2}$ are known as the 'positional boundary conditions' and 'derivative boundary conditions' respectively. ${ }^{15}$ Note that the surface patch will not necessarily pass through the curves which define the derivative boundary conditions.

\section{Interactive Parameterization of Geometry Model}

In this work the definition of the shape geometry is carried out using the PDE parameter model discussed in Ugail et $a l,{ }^{15}$ where the parameterized boundary curves are used to define the shape of the surface. Essentially, this parameterization is defined in such a way that linear transformations, such as translation, rotation and dilation, of the boundary curves can be carried out interactively. The result of this is that the designer is presented with tools which enable him/her to create and modify the geometry an intuitive manner.

For convenience, the parameterization on the boundary curves is denoted using the notation $c_{k P_{i}}$ $(k=1,2),(i=x, y, z)$. Here $c$ indicates the type of curve, with the letter $p$ denoting the position curves and the letter $d$ denoting the derivative curves. The index $k$ ranges from 1 to 2 corresponding to the $u=0$ and $u=1$ boundary edges (respectively) of the surface. The letter $P$ denotes the type of parameter: $T$ for a translation, $R$ for a rotation and $D$ for a dilation. Finally the letter $i$ denotes the coordinate directions relevant to a particular type of parameter. Adjustments to the values of these parameters along with the value of $a$ in equation (5) can be used to create and manipulate complex geometries.

As mentioned earlier, the effect of these parameters on the surface shape is easy to appreciate. Table 1 shows the values of the chosen parameters for $d=1$ for the surface shown in Fig. 1 .

In order to show the effect of the design parameters we now choose a different set of values for the parameters for the boundary $d=1$ of the surface shown in Fig. 1. The new values chosen for the parameters are shown in Table 2 and the resulting surface is shown in Fig. 2. Note the value of the $a$ for the surface shown in Fig. 2 is the same as that shown in Fig. 1, i.e. $a=1.0100$. Essentially, the new values of the parameters produced a dilation followed by a translation which is followed by a rotation of the boundary curve $d=1$. The parameters introduced on the boundary curves are varied 


\begin{tabular}{ll}
\hline parameter & value \\
\hline$d_{1 T_{x}}$ & 0.000 \\
$d_{1 T_{y}}$ & -0.850 \\
$d_{1 T_{z}}$ & 0.000 \\
$d_{1 D_{x}}$ & 0.771 \\
$d_{1 D_{y}}$ & 0.792 \\
$d_{1 D_{z}}$ & 0.000 \\
$d_{1 R_{x}}$ & 3.138 \\
$d_{1 R_{y}}$ & 0.000 \\
$d_{1 R_{z}}$ & 0.000 \\
\hline
\end{tabular}

Table 1 Values for the design parameters for the boundary $d=1$ of the surface shown in Fig. 1

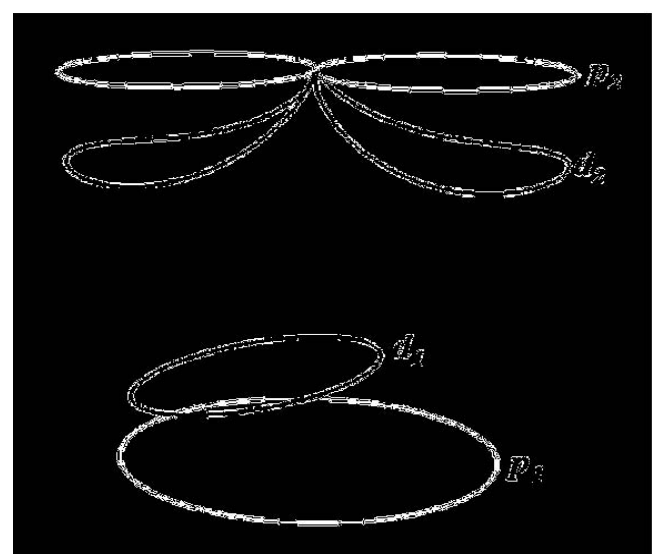

(a)

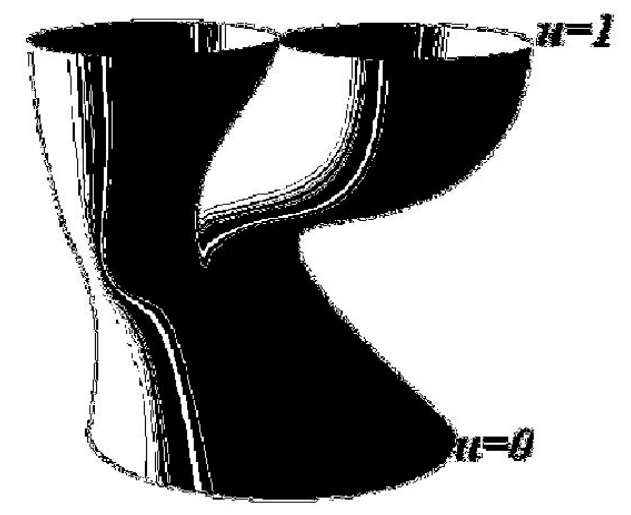

(b)

Fig. 2 The effect on the shape of the surface by changing the design parameters corresponding to the boundary $d=1$. (a) The boundary curves. (b) The corresponding PDE surface patch

\begin{tabular}{lc}
\hline parameter & value \\
\hline$d_{1 T_{x}}$ & -0.310 \\
$d_{1 T_{y}}$ & -0.850 \\
$d_{1 T_{z}}$ & 0.000 \\
$d_{1 D_{x}}$ & 0.371 \\
$d_{1 D_{y}}$ & 0.792 \\
$d_{1 D_{z}}$ & 0.000 \\
$d_{1 R_{x}}$ & 3.138 \\
$d_{1 R_{y}}$ & 0.000 \\
$d_{1 R_{z}}$ & 0.220 \\
\hline
\end{tabular}

Table 2 Values for the design parameters for the boundary $d=1$ of the surface shown in Fig. 2

using a graphical interface where the corresponding surface is visualized simultaneously. The spectral approximation method for solving the PDE, mentioned earlier, is fast enough for the surfaces to be created and manipulated in real time.

In order to build shapes corresponding to complex mechanical parts, more than one surface patch often needs to be joined together with common boundaries enabling to form a composite surface. ${ }^{13,14}$ The parametric model discussed above has been extended to cater for such composite bodies. For example, Fig. 3 shows a bifurcated port for a 2-stroke internal combustion engine. This composite surface has been created using three surface patches, where the final shape was produced by changing the values of the design parameters introduced onto the boundary curves which define the composite body.

\section{Design Optimization}

In this section we show how automatic design optimization can be carried out using the PDE parametric model discussed above. In particular, we show two examples taken from a practical setting, i.e., determination of the optimal shape of a single transfer port of a 2-stroke engine in order to reduce the size of the adverse pressure gradient when a gas mixture flows through the transfer port and the optimal design for strength of a "gravity loaded" structure by considering the shear stress within the structure.

As mentioned earlier, a typical design optimization problem can be thought of as maximizing or minimizing an objective function without violating a set of constraints. There exist a wide variety of methods for numerical optimization. The choice of a particular method is problem specific and involves considerations such as the computational cost of evaluating the function to be optimized,

4

American Institute of Aeronautics and Astronautics 


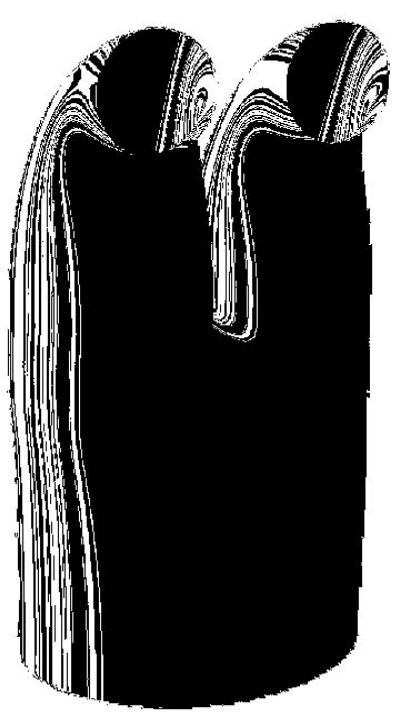

Fig. 3 A bifurcated port for a modern internal combustion engine created interactively.

and also the behavior of the function within the design space.

For the purpose of illustration we use the method of Simulated Annealing due to Kirkpatrick et al. ${ }^{8}$ Here we give a brief description of the Simulated Annealing and for more details the reader is referred to Laarhoven et al. ${ }^{10}$

\section{$\underline{\text { Simulated Annealing }}$}

Simulated Annealing is a stochastic optimization technique in which an initial design is chosen and the value for the objective function for that design is evaluated. A new design is then obtained by varying the design parameters, and the value of the objective function corresponding to this new design is then evaluated. If the new design has a better value in the objective function, the new design is accepted and becomes the current design. However, if the design does not lead to an improvement in the objective function, this new design may still be accepted with some probability. This probability is a function of a decreasing parameter called temperature, based on the analogy with the annealing of solids, given by:

$$
P_{\text {accept }}=e^{-\frac{\Delta C}{k T}}
$$

where $\Delta C$ is the non-dimensional change in the objective function due to the change in design parameters and $T$ is the current temperature. During the course of the optimization, the temperature starts out high and decreases with time. The design parameters are randomly varied leading to a random exploration of the objective function space. As the probability of accepting designs which do not produce improvements in the

\begin{tabular}{lccl}
\hline parameter & initial & min. & max. \\
$(k=1,2$ and $i=x, y, z)$ & & & \\
\hline$d_{k T_{i}}$ & 0.0 & -0.8 & 0.8 \\
$d_{k R_{i}}$ & 0.0 & -0.8 & 0.8 \\
$d_{k D_{i}}$ & 1.0 & 0.01 & 3.0 \\
$a$ & 0.5 & 0.01 & 9.0 \\
\hline
\end{tabular}

Table 3 Initial values and ranges for the parameters considered to obtain an inlet port.

objective function decreases, designs tend to get rejected, allowing the process to converge to an optimum. The temperature in a Simulated Annealing algorithm is controlled by an 'annealing schedule' which consists of an initial temperature, the number of design parameters involved and the overall number of temperatures.

\section{Optimization of a Transfer Port of an Internal} Combustion Engine

In this section we show the shape of the transfer port geometry could be optimized in order to improve its performance. For simplicity, we have used an objective function based on surface curvature rather than a measure based directly upon fluid dynamical properties so that the optimization takes place on a reasonable time-scale. Ideally one would like as a measure-of-merit a physical quantity such as the adverse pressure gradient in order to try to reduce flow separation. But obviously, this is time-consuming to calculate, and there is also the fact that it is difficult to verify the accuracy of calculations of this quantity.

Local curvature in the surface has a strong influence on separation. Here surface 'fairness' is a critical factor and in most cases, fair surfaces are desirable in designs where fluid separation is to be avoided. Therefore, for the purpose of illustration and to save time we have taken an objective function based on the local curvature of the surface as an alternative to a CFD calculation. In particular,

$$
f=\int\left(\kappa_{1}^{2}+\kappa_{2}^{2}\right) d s
$$

where $\kappa_{1}$ and $\kappa_{2}$ are the principal curvatures ${ }^{12}$ of the surface, was used as a 'measure-of-merit' that was minimized during the optimization.

For the sake of illustration, an initial geometry was produced using the methods discussed for the PDE surface. The initial geometry was created interactively, with the intention that its shape would be such that flow separation would be produced. This is shown in Fig. 4.

Using the PDE geometry parameterization model discussed earlier, the geometry of the port 


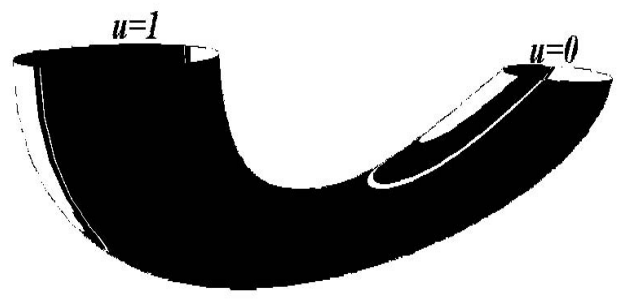

Fig. 4 Initial geometry of the transfer port.

\begin{tabular}{ll}
\hline parameter & optimal \\
\hline$d_{1 T_{y}}$ & 0.097 \\
$d_{1 D_{x}}$ & 0.771 \\
$d_{1 D_{y}}$ & 0.792 \\
$d_{1 D_{z}}$ & 0.721 \\
$d_{1 R_{z}}$ & 0.220 \\
$d_{2 T_{y}}$ & 0.165 \\
$d_{2 D_{x}}$ & 0.909 \\
$d_{2 D_{y}}$ & 0.918 \\
$d_{2 D_{z}}$ & 0.899 \\
$d_{2 R_{z}}$ & -0.172 \\
$a$ & 0.864 \\
\hline
\end{tabular}

Table 4 Final values of the parameters corresponding to the optimal design of the transfer port.

was parameterized and the initial values of the design parameters along with their ranges were then fed to the optimization routines. Table 3 shows the initial values and their range for the chosen parameters. Note that the range specified for each design parameter (by means of choosing a maximum and minimum) allows the parameters to be varied within the specified ranges enabling alternative shapes to be created within the design space automatically.

After several runs and experimenting with the number of parameters and their range, the optimal shape found is shown in Fig. 6. Fig. 5 shows some of the intermediate shapes of the transfer port obtained during optimization. The calculations were carried out on a PC workstation with a $400 \mathrm{MHz}$ processor and took about 20 minutes to complete.

The values of the parameters obtained for the optimal design is shown in Table 4. Note that the Table shows only the values for those parameters, which changed significantly during the optimization. The resulting optimal surface had a reduction in the chosen merit function of about $5 \%$.

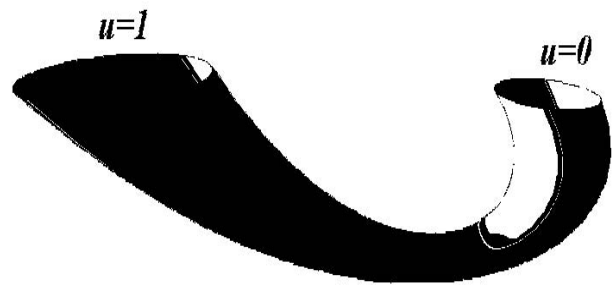

(a)

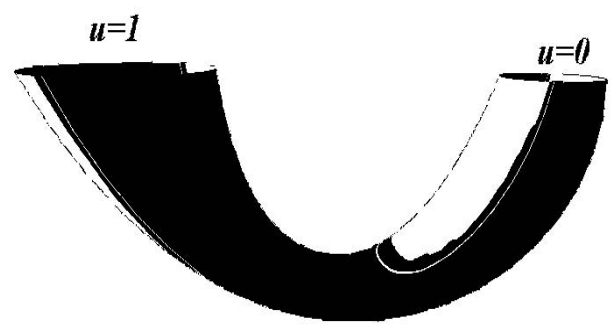

(b)

Fig. 5 Some of the Intermediate shapes of the transfer port obtained during optimization.

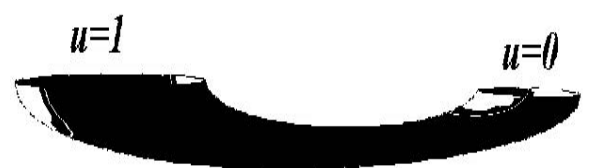

Fig. 6 Optimal geometry of the transfer port.

\section{Optimal Design of an Object for Strength}

Next we consider the optimal design of an object for strength based on the shear stress within the structure when the structure is gravity loaded. The example considered here is the optimal design for strength of the gravity loaded tower surface shown in Fig. 7(a). The design objective here is the minimization of the maximum shear stress. It is the excessive shear stress which can cause most damage to the material under consideration and therefore we are interested in minimizing this quantity. The objective function for this optimization therefore requires the calculation of the maximum shear stress that occurs in the solution for a particular loading. The shear stresses $\sigma_{x y}, \sigma_{x z}$ and $\sigma_{y z}$ are obtained by performing a finite element analysis of the structure. In this example, we carried out the analysis by assuming the structure to be an isotopic thin shell composed of aluminum. Details of how the finite element analysis for a shell structure can be carried out can be found in Niordson ${ }^{11}$ and Zienkiewicz. ${ }^{20}$ 


\begin{tabular}{llll}
\hline parameter & initial & min. & max. \\
\hline$d_{k T_{i}}$ & 0.0 & -1.2 & 1.2 \\
$(k=1,2$ and $i=x, z)$ & & & \\
$d_{1 T_{y}}$ & 0.0 & 0.0 & 1.2 \\
$d_{2 T_{y}}$ & 0.0 & -1.2 & 0.0 \\
$d_{k D_{i}}$ & 1.0 & 0.01 & 3.0 \\
$(k=1,2$ and $i=x, y, z)$ & & & \\
$a$ & 0.5 & 0.01 & 9.0 \\
\hline
\end{tabular}

Table 5 Initial values and ranges for the parameters considered to obtain an optimal design of the tower surface.

\begin{tabular}{ll}
\hline parameter & optimal \\
\hline$d_{1 D_{x}}$ & 0.942 \\
$d_{1 D_{y}}$ & 0.899 \\
$d_{1 D_{z}}$ & 0.979 \\
$d_{2 D_{x}}$ & 0.292 \\
$d_{2 D_{y}}$ & 0.310 \\
$d_{2 D_{z}}$ & 0.308 \\
$a$ & 0.754 \\
\hline
\end{tabular}

Table 6 Final values of the parameters corresponding to the optimal shape

The maximum shear stress $\sigma_{\max }^{p}$ occurring on any plane through a point $p$ is given by

$$
\sigma_{\max }^{p}=\max \left\{\left|\sigma_{x y}\right|,\left|\sigma_{x z}\right|,\left|\sigma_{y z}\right|\right\} .
$$

So the objective function is taken to be the maximum shear stress occurring in the whole structure, i.e.

$$
f=\underbrace{\max }_{\text {(all points) }}\left\{\sigma_{\max }^{p}\right\} .
$$

As with the previous example of the transfer port, a parameterization is introduced using the derivative boundary curves and the smoothing parameter is considered. Table 5 shows the initial values and the parameter ranges considered. Note that the parameterization considered in this case enables translations and dilations of the derivative boundary curves.

Once the PDE geometry is parameterized the optimizer routine is allowed to run. In this case the optimizer routine produces different configurations in parameter space and the geometry is varied.

In order to obtain a practical surface a volume constraint was also imposed, by means of a penalty function.

With the above settings the optimization provided favorable range of shapes of which Fig. 7(b)

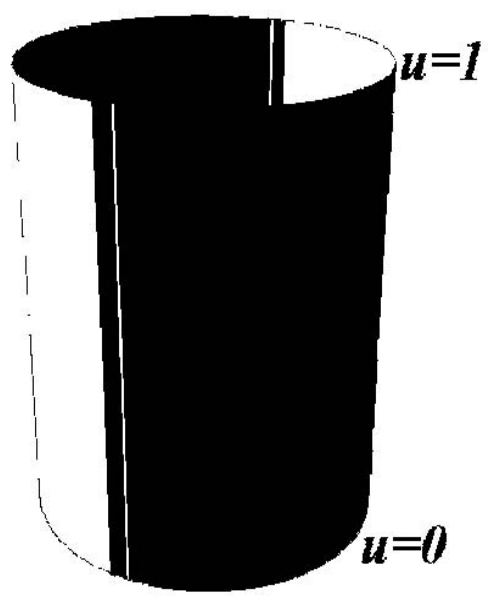

(a)

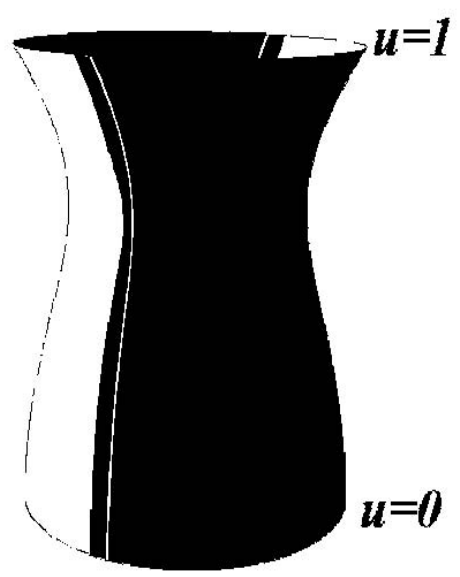

(b)

Fig. 7 Optimal design of an object for strength. (a) Initial shape of the tower surface (b) Optimal shape of the surfaces.

shows the shape of the optimal design. The values of the parameters obtained for this optimal design is shown in Table 6 . Note that the table only shows the values for those parameters whose values changed significantly during the optimization. The resulting optimal surface had a reduction in the maximum shear stress of about $6 \%$. Due to the extensive finite element analysis calculations, needed to calculate the value of the objective function for each iteration of the optimization, it took a little over 36 hours for the optimization to complete with the same computing hardware mentioned in the previous example.

\section{Conclusion}

In this paper we have demonstrated how this particular method for the description of complex geometries can be used to setup the shape opti- 
mization of practical surfaces. In particular, we have shown how the PDE geometry parameterization can be implemented to carry out automatic optimization within an interactive environment. Since the PDE method can describe complex shapes using the information contained at the boundaries (edges) of the surface patches we introduce an interactively defined parameterization on the boundary curves. The variation of these parameters leads to alternative geometries enabling an appropriate optimization routine to seek the geometry corresponding to a given merit function.

The examples we have discussed in this paper clearly shows the capability of the PDE parametric as a means of automatically varying the shape geometry of an object. It is our aim to further improve the parametric model so that automatic design optimization of complicated practical objects can be carried out efficiently.

\section{Acknowledgments}

The authors wish to acknowledge the support of EPSRC grant GR/L05730 ("The efficient parameterization of mechanical parts").

\section{References}

${ }^{1}$ Bloor, M.I.G., and Wilson, M.J., "Generating blend surfaces using partial differential equations," ComputerAided Design, 21, 165-171, 1989.

${ }^{2}$ Bloor, M.I.G., and Wilson, M.J., "Using Partial Differential Equations to Generate Freeform Surfaces," Computer Aided Design, 22, 202-212, 1990.

${ }^{3}$ Bloor, M.I.G., and Wilson, M.J., "Spectral Approximations to PDE Surfaces," Computer-Aided Design, 28, 145-152, 1996.

${ }^{4}$ Cohen, M.Z., "Theory and Practise of Structural Optimisation," Structural Optimisation, 7, 20-31, 1994.

${ }^{5}$ Dekanski, C.W., Bloor, M.I.G., and Wilson, M.J., "The Generation of Propeller Blades Using the PDE Method," Journal of Ship Research, 39, 108-116, 1995.

${ }^{6}$ Francavilla, A., Ramakrishanan, C.V., and Zienkiewicz, O.C.Z., "Optimisation of Shape to Minimise Stress Concentration," Journal of Strain Analysis, 10, 63-70, 1975.

${ }^{7}$ Imam, M.H., "Three-Dimensional Shape Optimisation," International Journal for Numerical Methods in Engineering, 18, 661-673, 1982.

${ }^{8}$ Kirkpatrick, S., Gellat, Jr., C.D., and Vecchi, M.P., "Optimisation by Simulated Annealing," Science, 220, (4598), 671-680, 1983.

${ }^{9}$ Kristensen, E.S., and Madsen, N.F., "On the Optimal Shape of Fillets in Plates Subject to Multiple InPlane Loading Cases," International Journal for Numerical Methods in Engineering, 10, 1007-1019, 1976.

${ }^{10}$ Laarhoven, P.J.M., van, and Aarts, E.H.L., Simulated Annealing: Theory and Applications. Mathematics and its Applications, D. Reidel Publishing Company, Dordrecht, Holland, 1987.

${ }^{11}$ Niordson, F.I., Shell Theory. Elsevier Science Publishers, Amsterdam, 1985.

${ }^{12}$ Struik, D., Lectures on Classical Differential Geometry. Addison-Wesley Pub. Co., Reading, Massachusetts, 1961.
${ }^{13}$ Ugail, H., Bloor, M.I.G. and Wilson, M.J., "On Interactive Design Using the PDE method," Mathematical Methods for Curves and Surfaces II, (eds) M. Dæhlen, T. Lyche, and L. L. Schumaker, Vanderbilt University Press Nashville TN, 493-500, 1998.

${ }^{14}$ Ugail, H., Bloor, M.I.G., and Wilson, M.J., "Techniques for Interactive Design Using the PDE Method," ACM Transactions on Graphics, 18, (2), 195-212, 1999.

${ }^{15}$ Ugail, H., Bloor, M.I.G., and Wilson, M.J., "Manipulations of PDE Surfaces Using an Interactively Defined Parameterisation," Computers and Graphics, 24, (3), 525534, 1999.

${ }^{16}$ Vida, J., Martin, R.R., and Varady, T., "A Survey of Blending Methods that use Parametric Surfaces," Computer-Aided Design, 26,(5), 341-365, 1994.

${ }^{17}$ Yao, T.M., and Choi, K.K., "3-D Shape Optimal Design and Automatic Finite Element Regridding," International Journal for Numerical Methods in Engineering, 28, 369-384, 1989.

${ }^{18}$ Yoo, Y.M., Haug, E.J., and Choi, K.K., "Shape Optimal Design of an Engine Connecting Rod," ASME Journal of Mechanisms, Transmission and Automation in Design, 106, 415-419, 1984.

${ }^{19}$ Zienkiewicz, O.C.Z., Campbell, J.S., "Shape Optimisation and Sequential Linear Programming," In Gallagher, R.H., and Zienkiewicz, O.C.Z., (Eds.), Optimal Structural Design, Wiley, London, pp. 109-126, 1973.

${ }^{20}$ Zienkiewicz, O.C.Z., The Finite Element Method. McGraw-Hill, New York, 1977. 